\title{
Effective gravity from a quantum gauge theory in Euclidean spacetime
}

\author{
R F Sobreiro and V J Vasquez Otoya \\ CBPF, Centro Brasileiro de Pesquisas Físicas, Rua Xavier Sigaud 150, 22290-180 Urca, \\ Rio de Janeiro, Brazil \\ E-mail: sobreiro@cbpf.br and vjose@cbpf.br
}

Received 22 May 2007, in final form 12 August 2007

Published 25 September 2007

Online at stacks.iop.org/CQG/24/4937

\begin{abstract}
We consider a $S O(d)$ gauge theory in a Euclidean $d$-dimensional spacetime, which is known to be renormalizable to all orders in perturbation theory for $2 \leqslant d \leqslant 4$. Then, with the help of a spacetime representation of the gauge group, the gauge theory is mapped into a curved spacetime with a linear connection. Furthermore, in this mapping the gauge field plays the role of the linear connection of the curved spacetime and an effective metric tensor arises naturally from the mapping. The obtained action, being quadratic in the Riemann-Christoffel tensor, at first sight, spoils a gravity interpretation of the model. Thus, we provide a sketch of a mechanism that breaks the $S O(d)$ color invariance and generates the Einstein-Hilbert term, as well as a cosmological constant term, allowing an interpretation of the model as a modified gravity in the Palatini formalism. In that sense, gravity can be visualized as an effective classical theory, originated from a well-defined quantum gauge theory. We also show that, in the four-dimensional case, two possibilities for particular solutions of the field equations are the de Sitter and anti-de Sitter spacetimes.
\end{abstract}

PACS numbers: 11.10.Gh, 11.10.Kk, 11.15.-q, 11.30.-j

\section{Introduction}

Since the beginning of the last century, many attempts to give gravity a quantum description have been made. However, a complete consistent quantum theory of gravity is still absent. In particular, attempts to quantize general relativity have failed since it is a nonrenormalizable theory where higher order derivative counterterms already appear at one-loop quantum corrections [1-3]. In order to solve this problem, gravity has been described by several alternative approaches. Examples include the higher derivative gravities [4]; the loop quantum gravity (LQG) [5, 6]; the Einstein-Cartan (EC) gauge formalism [7, 8]; and the interpretation that gravity would be an effective theory originated from more general theories such as 
superstring theories $[9,10]$. It is important to highlight though that it is not our intention to give a complete description on these issues, for that we refer to the cited bibliography and references therein.

To discuss quantum aspects of gravity, let us take into consideration the pure quantum mechanical point of view. It is reasonably unanimous that quantum physics is the fundamental theory while classical physics is a particular case of the quantum theory. From this point of view, quantization methods employed to achieve a quantum model from a classical model are just ansätze or algorithms that might not always work. A renowned example is the general dissipative system. This is the main quantum mechanics point, implying that the word quantize should not be taken as a fundamental principle [11, 12]. In quantum field theory, the picture is no different. The quantization methods are just ansätze successfully utilized to obtain a quantum theory from a classical model. The starting point would be the fundamental quantum theory from where, in some suitable limits, one recovers the classical equivalent theory. This might be the reason why gravity resists being quantized by the usual quantization methods, requiring then a more fundamental understanding of quantum theory.

Thus, based on the fundamental character of the quantum theory and on the success of gauge theories when utilized to describe the fundamental interactions, we opt, in this work, for the idea that gravity can be visualized as an effective theory originated from a simple, non-Abelian, four-dimensional Euclidean quantum gauge field theory. To be more specific, we start our investigation on the proposed idea by considering a $S O(d)$ gauge theory in a Euclidean $d$-dimensional spacetime ${ }^{1}$. Under this prescription, the graviton is simply defined as the gauge field, $A_{i}^{a}$. We recall that an essential ingredient of this description is that there is no curved space in the quantum sector of the theory. In fact, the Euclidean spacetime is a motivation to define the path integral allowing a useful consistent scenario to perform the quantum computations.

Once we have established the properties of the theory at the quantum level, we can relate it with a curved spacetime through a mapping from the Euclidean gauge theory to a dynamical spacetime with $G L(d, \mathbb{R})$ symmetry. For that, one can use a representation of the $S O(d)$ gauge group borrowing the Euclidean spacetime indices, then the group index $a$ can be represented by a pair of antisymmetric spacetime indices, $A_{i}{ }^{j k}=-A_{i}{ }^{k j}$. In this mapping, the Euclidean space is identified with a curved space through transformation matrices $e_{i}^{\mu}$, which are related to an effective metric tensor $g$. The gauge field is mapped into a linear connection of the spacetime $A_{i j}{ }^{k} \mapsto \Gamma_{\mu \nu}{ }^{\alpha}$ which is independent of the metric tensor. Thus, the Euclidean gauge theory is mapped into a dynamical spacetime in the Palatini formalism. The original action, $\int \mathbf{F}^{2}$, is mapped into an action quadratic in the Riemann tensor $\int \sqrt{g} \mathbf{R} \cdot \mathbf{R}$.

In order to relate the dynamical curved space with gravity, it would be useful to generate the Einstein-Hilbert (EH) term in the action. However, the term $\mathbf{R} \cdot \mathbf{R}$ still carries the global gauge symmetry of the original gauge theory while the EH term, $R=R_{\mu \nu}{ }^{\mu \nu}=\operatorname{Tr} \mathbf{R}$, breaks color invariance. Thus, we provide a sketch of a color symmetry breaking mechanism which generates the EH term. The first feature of the color symmetry breaking is the appearance of physical spin-2 excitations originating from the antisymmetry structure of the color indices. Before the breaking, these excitations were hidden behind the color symmetry and thus were unphysical. It is important to know that this mechanism is employed in the quantum sector of the gauge theory, i.e., the mapping is only performed after the mechanism is worked out. This detail ensures the existence of a framework for well-defined quantum computations, as a Euclidean QFT is. An extra feature of the mechanism is that a cosmological constant (CC) appears naturally in the resulting action. We remark at this point, and it will be pointed

1 Here we are considering a $d$-dimensional space just for generality purposes, the main focus being the case $d=4$. 
out further, that this mechanism is merely a possible sketch. In fact, further investigation on the formalization of the present mechanism (or the other) is needed and is currently under investigation [13].

Finally, once the previous mechanism is worked out, the mapping can be performed. The obtained action for the dynamical spacetime is now constituted by a quadratic term in the Riemann tensor, the $\mathrm{EH}$ term and the $\mathrm{CC}$ term. This new action can now be related with gravity, in the Palatini formalism. In the particular case of four dimensions, the equations of motion provide the de Sitter spacetime $\left(d S_{4}\right)$ or the anti-de Sitter $\left(A d S_{4}\right)$ as the fundamental spacetime.

This work is organized as follows. In section 2, we will show how a $S O(d)$ gauge model, in the $d$-dimensional Euclidean spacetime, can be mapped into a dynamical curved space. The resulting dynamical space-time enjoys $G L(d, \mathbb{R})$ symmetry and $S O(d)$ gauge symmetry (color invariance). In section 3 , we will argue that, to obtain an effective gravity theory from the previous method, a color symmetry breaking mechanism is required. In section 4, a color symmetry breaking mechanism will be sketched and, due to this mechanism, the EH term is generated together with the CC. Also in this section we will discuss the resulting classical field equations. Finally, the conclusions will be displayed in section 5 .

\section{From $S O(d)$ gauge theory to the dynamical curved spacetime}

We start this section with a small review of the definitions, notations and properties of the $S O(d)$ gauge theory.

\subsection{Generalities of the $S O(d)$ gauge theory}

Let us consider the following principal bundle

$$
\mathcal{P} \equiv\left\{S O(d) ; \mathbb{R}^{d} ; \mathcal{A}\right\}
$$

where $S O(d)$ is the group of orthogonal matrices characterizing the fiber and the structure group of the fiber. There are $D=d(d-1) / 2$ generators of this group which we label by $\lambda^{a}, a \in\{1,2, \ldots, D\}$. The algebra is a typical Lie algebra,

$$
\left[\lambda^{a}, \lambda^{b}\right]=f^{a b c} \lambda^{c},
$$

where $f^{a b c}$ represents the structure constants of the group. Also, we denote the elements of the group by $u=\mathrm{e}^{\omega^{a} \lambda^{a}}$. The total space of the principal bundle (1) is chosen to be a Euclidean $d$-dimensional space, $\mathbb{R}^{d}$, which enjoys a global $O(d)$ symmetry. The set $\mathcal{A}$ is the space of the algebra-valued connections, the base space ${ }^{2}$,

$$
A_{i}=A_{i}^{a} \lambda^{a} \text {. }
$$

The homeomorphisms of the principal bundle are characterized by the gauge transformations

$$
A_{i} \rightarrow A_{i}+u^{\dagger} D_{i} u
$$

where the covariant derivative is just

$$
D_{i} \cdot=\partial_{i} \cdot+\left[A_{i}, \cdot\right]
$$

Infinitesimal gauge variations are then defined as

$$
\delta A_{i}=D_{i} \omega .
$$

2 The first part of the Latin alphabet, $\{a, b, c, \ldots, h\}$, labels the group indices while the second part, $\{i, j, k, \ldots, z\}$, will be used to label the spacetime coordinates. 
The gauge invariant action is

$$
S_{\mathrm{YM}}=\frac{1}{4 \kappa^{2}} \operatorname{Tr} \int \mathrm{d}^{d} x F_{i j} F^{i j},
$$

where the field strength (fiber curvature) is given by

$$
F_{i j}=F_{i j}^{a} \lambda^{a}=\partial_{i} A_{j}-\partial_{j} A_{i}+\left[A_{i}, A_{j}\right] .
$$

The quantity $\kappa$ is the coupling constant.

To quantize the model, a gauge fixing is usually needed. Here we choose the simplest covariant gauge, the Landau gauge $\partial^{i} A_{i}=0$. With this purpose, we add to the gauge invariant action (7) a gauge-fixing term

$$
S=S_{\mathrm{YM}}+S_{\mathrm{gf}}
$$

where

$$
S_{\mathrm{gf}}=\operatorname{Tr} \int \mathrm{d}^{d} x\left(b \partial^{i} A_{i}+\bar{c} \partial^{i} D_{i} c\right)
$$

The fields $c=c^{a} \lambda^{a}$ and $\bar{c}=\bar{c}^{a} \lambda^{a}$ are, respectively, the Faddeev-Popov ghost and antighost fields while $b=b^{a} \lambda^{a}$ is the Lautrup-Nakanishi field which plays the role of a Lagrange multiplier enforcing the Landau gauge condition. The action (9) enjoys renormalizability, i.e., the model is consistent at the quantum level. In fact, as discussed in appendix A, the renormalizability of action (9) can be established to all orders in perturbation theory for $2 \leqslant d \leqslant 4$

After gauge fixing, a global $S O(d)$ gauge symmetry survives, the so-called color invariance. This invariance is characterized by the non-observational character of the group indices. In terms of a Ward identity, color invariance is described by

$$
\int \mathrm{d}^{d} x\left(\left[A_{i}, \frac{\delta S}{\delta A_{i}}\right]+\left[c, \frac{\delta S}{\delta c}\right]+\left[\bar{c}, \frac{\delta S}{\delta \bar{c}}\right]+\left[b, \frac{\delta S}{\delta b}\right]\right)=0 .
$$

\subsection{Spacetime representation}

Unless the contrary is said, we will not consider the gauge-fixing term for the rest of the paper. Thus, from now on, we are dealing with the gauge invariant action (7), and global color invariance is now described by

$$
\int \mathrm{d}^{d} x\left[A_{i}, \frac{\delta S_{\mathrm{YM}}}{\delta A_{i}}\right]=0 .
$$

First, we will explore the fact that the $S O(d)$ group, in a Euclidean spacetime, can borrow the spacetime structure to fix a representation of the group. For this, we observe that the dimension of the total space $\mathbb{R}^{d}$ coincides with the Casimir $d$ of the group $S O(d)$, which means that the generators can be represented as a set of $(d \times d)$-matrices. Also, the group dimension, $D$, coincides with the number of independent elements of an antisymmetric $(d \times d)$-matrix. These properties allow a unique representation of the gauge group where the generators are in the form of matrices labeled by spacetime indices,

$$
\lambda^{a} \mapsto \lambda^{i j}, \quad f^{a b c} \mapsto f^{i j k l m n},
$$

where

$\lambda^{i j}=-\lambda^{j i}, \quad f^{i j k l m n}=-\frac{1}{2}\left[\left(\delta^{i l} \delta^{m j}-\delta^{j l} \delta^{m i}\right) \delta^{k n}+\left(\delta^{j k} \delta^{i m}-\delta^{i k} \delta^{m j}\right) \delta^{l n}\right]$.

Thus

$$
A_{i}^{a} \lambda^{a}=A_{i j}{ }^{k} \lambda^{j}{ }_{k}, \quad F_{i j}^{a} \lambda^{a}=F_{i j k}{ }^{l} \lambda^{k}{ }_{l} .
$$


In the above described representation, the action (7) reads

$$
S_{\mathrm{YM}}=\frac{1}{4 \kappa^{2}} \int \mathrm{d}^{d} x F_{i j k}{ }^{l} F_{l}^{i j k}
$$

where

$$
F_{i j k}{ }^{l}=\partial_{i} A_{j k}{ }^{l}-\partial_{j} A_{i k}{ }^{l}+A_{i m}{ }^{l} A_{j k}{ }^{m}-A_{j m}{ }^{l} A_{i k}{ }^{m} .
$$

Thus, we are dealing with a gauge theory, in a Euclidean spacetime with a representation where the color indices are represented by spacetime indices.

As previously pointed out, the color invariance has a fundamental role in this paper; therefore, for further use, let us write the color symmetry Ward identity in the spacetime representation:

$$
\int \mathrm{d}^{d} x\left(A_{k i}{ }^{n} \delta_{m j}-A_{k j}{ }^{n} \delta_{m i}\right) \frac{\delta S_{\mathrm{YM}}}{\delta A_{k m}{ }^{n}}=0 .
$$

\subsection{Pseudo-vielbein and curved spacetime}

Looking at the field strength expression in (17), one can recognize an extreme similarity with a Riemann-Christoffel tensor of a curved spacetime. Thus, we can absorb the gauge field as a geometrical structure of the spacetime and end up in an effective geometrical theory, completely equivalent to a gauge theory. In fact, we can perform a mapping from the Euclidean space to a curved space $^{3}$,

$$
\left\{x_{i}\right\} \mapsto\left\{x_{\mu}, x^{\mu}\right\},
$$

by introducing the pseudo-vielbein and its inverse

$$
e_{\mu}^{i}=\frac{\partial x^{i}}{\partial x^{\mu}}, \quad \bar{e}_{i}^{\mu}=\frac{\partial x^{\mu}}{\partial x^{i}} .
$$

Thus

$$
\mathrm{d} x^{i}=e_{\mu}^{i} \mathrm{~d} x^{\mu}, \quad \mathrm{d} x^{\mu}=\bar{e}_{i}^{\mu} \mathrm{d} x^{i} .
$$

The name pseudo-vielbein ${ }^{4}$ is employed here due to the fact that $e$ is not the vielbein of the EC theory of gravity [7, 8], as will become evident in what follows. In that sense, the flat space is completely independent of the curved space. Differently from the EC formalism, the p-vielbein does not define a reference frame which travels tangently on the curved space. Here, the p-vielbein is a point-to-point identification of the two spaces which have no direct geometrical relation. We will return to this issue soon.

The requirement of metric preservation in both spaces implies the existence of a symmetric metric tensor $\left\{g_{\mu \nu}, g^{\mu \nu}\right\}$, in fact the following relations hold,

$$
e_{\mu}^{i} e_{i v}=g_{\mu \nu}, \quad \bar{e}_{i}^{\mu} \bar{e}_{\mu}^{j}=\delta_{i}^{j}, \quad e_{i}^{\mu} \bar{e}_{\mu}^{j}=\delta_{i}^{j}, \quad e_{i}^{\mu} \bar{e}_{v}^{i}=\delta_{v}^{\mu},
$$

where the first two are related to the metric invariance in both spaces. In that sense the p-vielbein is equivalent to the usual vielbein, which justifies the name. However, here $e$ is essentially a mapping field.

For the ordinary derivative and for the gauge field, the mapping occurs according to

$$
\partial_{i}=\bar{e}_{i}^{\mu} \partial_{\mu}, \quad A_{i j}{ }^{k}=\bar{e}_{i}^{\mu} \bar{e}_{j}^{\nu} e_{\alpha}^{k} \Gamma_{\mu \nu}{ }^{\alpha}-\bar{e}_{i}^{\mu} e_{\nu}^{k} \partial_{\mu} \bar{e}_{j}^{\nu} .
$$

The quantity $\Gamma$ is identified with the connection of the curved space.

3 The Greek indices label the coordinates of the curved space time.

4 For economical purposes we will call the pseudo-vielbein simply by p-vielbein. 
Now we return to the geometrical properties of the p-vielbein. In the EC formalism, the vielbein connects the curved space with a tangent space. This association implies that the partial derivatives do not commute in the flat space while the derivatives in the curved space do commute. In our formalism, the original flat spacetime is not a tangent space of the curved space. They are essentially independent spaces, modulo the mapping through $e_{i}^{\mu}$. Thus, the partial derivative commutes in the Euclidean space as well as in the curved space,

$$
\left[\partial_{i}, \partial_{j}\right]=0, \quad\left[\partial_{\mu}, \partial_{\nu}\right]=0 .
$$

These commutation rules imply that the non-holonomicity coefficients of both spaces vanish. Consequently, the p-vielbein behaves exactly as a coordinate matrix transformation.

Finally, we wish to make it clear that there is no a priori proof of the existence of consistent (invertible) transformations $e_{i}^{\mu}$. For now, we naively assume that $e_{i}^{\mu}$ exists and is invertible. Nevertheless, this assumption will be confirmed a posteriori when we solve the field equations in section 4.4. Essentially, a kind of proof of the existence of an invertible $e_{i}^{\mu}$ would rely on solving the field equations in the curved spacetime and find an invertible metric tensor as a consistent solution.

\subsection{Action for the dynamical curved space}

A problem to be faced at this point is that we must generate a scalar action on the curved space, which means that $F$, in the curved space, should be a tensor. Substituting (23) in (17) and making use of the relations (24), we find

$$
F_{i j k}{ }^{l}=\bar{e}_{i}^{\mu} \bar{e}_{j}^{\nu} \bar{e}_{k}^{\alpha} e_{\beta}^{l} R_{\mu \nu \alpha}^{\beta},
$$

where $R_{\mu \nu \alpha}^{\beta}$ is a four-rank tensor in the form

$$
R_{\mu \nu \alpha}^{\beta}=\partial_{\mu} \Gamma_{\nu \alpha}^{\beta}-\partial_{\nu} \Gamma_{\mu \alpha}^{\beta}+\Gamma_{\mu \gamma}^{\beta} \Gamma_{\nu \alpha}^{\gamma}-\Gamma_{\nu \gamma}^{\beta} \Gamma_{\mu \alpha}^{\gamma},
$$

recognized as the Riemann-Christoffel tensor. At the action (16), this transformation results in

$$
S_{Y M}^{\mathrm{e}}=\frac{1}{4 \kappa^{2}} \int \mathrm{d}^{d} x \mathrm{e} R_{\mu \nu \alpha}{ }^{\beta} R_{\beta}^{\mu \nu \alpha},
$$

where $e=\operatorname{det} e_{\mu}^{i}=\sqrt{-\operatorname{det} g_{\mu \nu}}$ comes from the Jacobian of the transformation (21). The action (27) displays color invariance under the $S O(d)$ group characterized by the following functional identity

$$
\int \mathrm{d}^{d} x\left(\Gamma_{\mu \nu}{ }^{\alpha} \delta_{\beta \gamma}-\Gamma_{\mu \gamma}{ }^{\alpha} \delta_{\beta \nu}\right) \frac{\delta S_{\mathrm{YM}}}{\delta \Gamma_{\mu \beta}{ }^{\alpha}}=0 .
$$

Furthermore, it is clear that (27) enjoys $G L(d, \mathbb{R})$ symmetry, not at the gauge sector, but in the curved spacetime itself. What does this mean? It means that we started from a renormalizable gauge theory. Due to the possibility of the spacetime representation, the classical theory is then mapped into a curved space with a linear connection. The gauge field turns into the linear connection and an effective metric tensor arises from the p-vielbein.

\subsection{On-shell field equations}

The action (27) describes the dynamics of a curved spacetime. The fundamental field being the connection $\Gamma$. The explicit form of $\Gamma$, which dictates the class of geometry we are dealing 
with, is unknown until now. Thus, at the classical level, the field equations of the connection are just

$$
-\mathcal{D}_{\mu} R_{\beta}^{\mu \nu \alpha}-2\left[\frac{d}{2} Q_{\mu}+T_{\mu \kappa}{ }^{\kappa}\right] R_{\beta}^{\mu \nu \alpha}+T_{\mu \kappa}{ }^{\nu} R_{\beta}^{\mu \kappa \alpha}=0,
$$

where

$$
Q_{\mu}=\frac{1}{d} g^{\alpha \beta} \mathcal{D}_{\mu} g_{\alpha \beta}
$$

is the Weyl convector and

$$
T_{\mu \nu}^{\alpha}=\frac{1}{2}\left(\Gamma_{\mu \nu}^{\alpha}-\Gamma_{\nu \mu}^{\alpha}\right)
$$

is the torsion tensor. The covariant derivative $\mathcal{D}$ is the usual covariant derivative of a general curved spacetime with the linear connection $\Gamma$, which is different from the definition of the covariant derivative (5).

Evidently, the p-vielbein constitutes a field and it will have its respective field equation. However, it is important to keep in mind that the fundamental field here is the connection which has well-defined quantum properties. The p-vielbein is a pure classical field. The classical field equations of the connection are the usual classical limit selecting the main contribution to the path integral. On the other hand, the field equations for the p-vielbein are interpreted as a minimization principle related to the vacuum energy stability. In fact, there are several possible solutions to $e$. Nevertheless, through the field equations, we select the p-vielbein which makes the vacuum energy stable. In principle, it is just a matter of minimal principles. Thus, the variation of the action (27) with respect to the metric tensor provides ${ }^{5}$

$$
\frac{1}{2} g^{\mu \nu} R^{\sigma \rho \alpha \beta} R_{\sigma \rho \alpha \beta}-R_{\alpha \beta \gamma}^{\mu} R^{\nu \alpha \beta \gamma}-R_{\alpha \beta \gamma}{ }^{\mu} R^{\alpha \beta \gamma \nu}=0 .
$$

Now, contracting the equation for the p-vielbein with $g_{\mu \nu}$ one finds

$$
\frac{(d-4)}{2} R^{\sigma \rho \alpha \beta} R_{\sigma \rho \alpha \beta}=0 .
$$

We recall that the geometry involved is determined by the field equations (29) and (32). The solution of these equations will provide the geometrical properties of the curved space. The curved space is simply a space with a single linear connection, which generalizes several geometries such as the Riemannian geometry and the Einstein-Cartan geometry. From that point of view, we are dealing with the Palatini formalism, the so-called metric-affine formulation. The difference is that action (27) no longer describes gravity. In the next section, we start the discussion whether the presented formalism can be related to a gravity theory or not.

Exploiting the field equations (29), (32) and (33) for $d \neq 4$, we observe that equation (33) implies that $R^{\sigma \rho \alpha \beta}=0$, which is also a solution of (29) and (32). In other words, the case $d \neq 4$ is trivial when matter is not being considered.

For the case $d=4$, equation (33) is satisfied for any non-vanishing curvature. In this case, the nontrivial solutions of (29) are allowed and nontrivial curved spaces show up. Obviously, this phenomenon occurs due to the nonlinear character of the action (27). Physically, this character is associated to the self interaction of $\Gamma$.

\section{Intermediate discussion}

We wish to relate the above described theory with gravity through the following point of view.

5 The same equation can be achieved by varying the action (27) with respect to the p-vielbein. 
Quantum gravity would be described by the action (7) in Euclidean spacetime, which is unitary and renormalizable. In this hypothesis, the gauge field (3) is interpreted as the graviton. Thus, at very high energies gravity is simply a renormalizable quantum gauge theory in Euclidean spacetime, where a spin-1 gauge field plays the role of the graviton.

On the other hand, at low energies, the theory can be described by a deformed spacetime where the gauge field is visualized as a linear connection. In that regime an effective metric tensor arises due to the presence of the p-vielbein. Thus, action (27) can be regarded as a kind of effective theory of gravity. In that sense, classical gravity is no longer a fundamental theory. The deformation of the spacetime occurs from quantum effects of a gauge theory as well as the covariance principle of general relativity.

We remark that the present approach to treating gravity is essentially different of the Einstein-Cartan formalism [7, 8]. In the EC formalism, there is an initial assumption of the existence of a curved space as the fundamental space. The tangent flat space is used in order to define the spin connection which allows the introduction of fermion fields with the help of the vielbein. However, the formalism still lacks renormalizability once the EH term is taken as the fundamental action for gravity. In our prescription, the fundamental theory is a consistent Euclidean quantum gauge theory of spin-1 fields which, for a certain limit, is equivalent to a pure dynamical curved spacetime where no reference to a tangent space or a spin connection is made. Thus, the curved space is a direct consequence of the dynamics of the gauge theory and not the contrary. Furthermore, the original flat space is not a tangent space of the curved space, as is evident from (24).

This idea turns on essentially three issues to be faced. First of all: where is the EH term $\propto R=R_{\nu \mu}^{\mu \nu}$, which ensures the relation of a theory with gravity? It is easy to see that this question directly hits the color symmetry of action (27). In fact, the EH term would require contraction between group indices and spacetime indices, which breaks color invariance.

The second point concerns the spin of the physical excitations. At the quantum level, the physical excitations carries spin-1, as any gauge theory of vector connection. Curiously, in the spacetime representation, spin-2 excitations also show up, due to the identification of the group indices with the spacetime indices. However, due to color symmetry, those are nonphysical excitations, which will never be observed. Thus, the question of the spin-2 physical excitations persists: would color invariance forbid the existence of spin-2 excitations at the physical sector of the theory?

Finally, the equivalence between actions (7) and (27), for now, due to color invariance, seems to be just a point of view, i.e., just another way to look at the nature. It happens because color invariance forbids the observational character of color indices. For example, in $R_{\mu \nu \alpha \beta}$ the last two indices are related to color invariance. Then, if one wishes to measure, directly, the curvature of the space time, it would never be successful, since the last two indices are non-observable. Therefore, the third question is: which physical effect pushes the theory to the action (27), characterizing the low energy regime of (7) in terms of an observable curved spacetime?

These questions are then closely related to the color symmetry of action (27). Furthermore, a color symmetry breaking mechanism seems to be necessary if one wishes to describe gravity with the above program. With this purpose, in the next section we will provide a sketch of a possible color symmetry breaking mechanism. We will show that this mechanism can generate the physical spin-2 excitations as well as the EH term. Furthermore, the same mechanism would dictate the vacuum of the theory, allowing a physical motivation for the mappings (21) and (23).

We would like to recall, at this point, that the above statements and what follows next only work for $2 \leqslant d \leqslant 4$, as is required by the renormalizability condition of the theory. 


\section{Color symmetry breaking and gravity}

For the beginning of this section, we forget the dynamical curved spacetime equivalent theory and turn back to the Euclidean theory (1).

\subsection{A background field}

Let us consider, again, the action (16). We suppose the existence of a background field $\Upsilon$ which generates the following field strength:

$$
F_{i j k}^{l}(\Upsilon)=m^{2}\left(\delta_{i}^{l} \delta_{j k}-\delta_{i k} \delta_{j}^{l}\right),
$$

where $m$ has the dimension of a mass. It is evident that the field strength renormalizes according to the renormalization of the gauge field and the coupling constant. Thus, a field strength for a background would require that $m$ renormalizes nontrivially according to the renormalization of the gauge field and the coupling constant. In fact, according to appendix B, the renormalization factor of $m^{2}$ is not independent and is given by

$$
Z_{m^{2}}=Z_{A} Z_{\kappa}^{2} \text {. }
$$

The condition (34) implies that $\Upsilon$ is a solution of the classical field equations, if one requires the following extra condition

$$
\Upsilon_{i}^{l i} \delta_{j k}-\Upsilon_{i k}^{i} \delta_{j}^{l}+\Upsilon_{k j}^{l}-\Upsilon_{k}^{l}{ }_{j}=0
$$

It is not difficult to see that equation (34) suggests that $\Upsilon$ is a singular configuration. For that, we write $\Upsilon$ as a pure gauge, which is just a gauge equivalent of the vacuum configuration $\Upsilon^{\prime}=0$,

$$
\Upsilon_{i k}^{l}=\partial_{i} \theta_{k}^{l}
$$

And then, considering the linear approximation of equation (34), one finds

$$
\left(\partial_{i} \partial_{j}-\partial_{j} \partial_{i}\right) \theta_{k}^{l}=m^{2}\left(\delta_{i}^{l} \delta_{j k}-\delta_{i k} \delta_{j}^{l}\right) .
$$

This equation establishes the singular nature of $\Upsilon$ since $\theta$ is clearly singular. An explicit example can be found in [15], where $\Upsilon$ is associated with flux tubes in an Abelian superconductor.

\subsection{Color symmetry breaking}

From (34) we see that $\Upsilon$ explicitly breaks color invariance since it mixes color and spacetime indices. As a consequence it will turn the color indices into observable spacetime indices. On the other hand, one might argue on the physical consequences of the background in the sense that the field strength, in the form (34), is an exclusive solution of the spacetime representation. This means that this effect can be described exclusively in the spacetime representation. To answer this question, we can take a look on the Noether current for global color symmetry,

$$
j_{p i j}(A)=\frac{\delta S_{Y M}}{\delta\left(\partial_{p} A_{k m}{ }^{n}\right)} \delta_{(i j)} A_{k m}{ }^{n},
$$

where, from (12),

$$
\delta_{(i j)} A_{k m}{ }^{n}=\delta_{m j} A_{k i}{ }^{n}-\delta_{m i} A_{k j}{ }^{n} .
$$

Thus

$$
j_{p i j}(A)=A_{k i}{ }^{n} F_{p k j}{ }^{n}-A_{k j}{ }^{n} F_{p k i}{ }^{n} .
$$


For the background we then have

$$
j_{p i j}(\Upsilon)=m^{2}\left(\Upsilon_{k i}{ }^{k} \delta_{j p}-\Upsilon_{k j}{ }^{k} \delta_{i p}+\Upsilon_{i j p}-\Upsilon_{j i p}\right) .
$$

Making use of condition (36), we achieve, identically,

$$
j_{p i j}(\Upsilon)=0
$$

Thus, the background configuration lies at the nonphysical sector of the theory. This means that the massless Goldstones states, associated with the color symmetry breaking, should decouple from the physical spectrum of the theory.

Obviously, considering the gauge-fixing term, (10), our conclusion remains the same since the difference is just a BRST exact term

$$
j_{p}{ }^{i j}(\Upsilon)=s[\mathcal{F}(\Upsilon, A, \bar{c}, c, b)],
$$

where $\mathcal{F}$ is a functional of the fields and the background. In (44) the BRST variation of the background is defined according to

$$
s \Upsilon_{k}^{a}=f^{a b c} \Upsilon_{k}^{b} c^{c},
$$

or, in the spacetime representation,

$$
s \Upsilon_{k i}^{j}=\Upsilon_{k i}{ }^{m} c^{j}{ }_{m}-\Upsilon_{k m}{ }^{j} c^{m}{ }_{i} .
$$

For the other fields the BRST transformations are given in (A.1).

\subsection{Effective action for the background}

Now, we write the gauge field as a perturbation around the background

$$
A_{i j}{ }^{k} \rightarrow \Upsilon_{i j}{ }^{k}+A_{i j}{ }^{k}
$$

Thus,

$F_{i j k}{ }^{l}(A) \rightarrow F_{i j k}{ }^{l}(A)+m^{2}\left(\delta_{i k} \delta_{j}^{l}-\delta_{i}^{l} \delta_{j k}\right)+\Upsilon_{i}{ }^{m}{ }_{k} A_{j m}{ }^{l}-\Upsilon_{i m}{ }^{l} A_{j}{ }^{m}{ }_{k}-\Upsilon_{j}{ }^{m}{ }_{k} A_{i m}{ }^{l}+\Upsilon_{j m}{ }^{l} A_{i}{ }^{m}{ }_{k}$.

To avoid terms depending on the background in (48) and, consequently, in the resulting action, we write $\Upsilon$ as $^{6}$

$$
\Upsilon_{i j}{ }^{k}=\mathcal{F}_{i j}{ }^{k}-D_{i j m}{ }^{k l} h_{l}^{m},
$$

in such a way that $\Upsilon$ is fixed in order to maintain the relation (34) while $\mathcal{F}$ and $h$ are arbitrary, and the covariant derivative is taken with respect the background itself, $D=D(\Upsilon)$. Thus, we can get rid of the $\Upsilon$ dependent term in (48) by making the smooth limit of the arbitrary functions $\mathcal{F} \leftrightarrow D \cdot h$ while $F(\Upsilon)$ remains fixed and $\Upsilon$ becomes small. Thus, we have taken the limit $\Upsilon \rightarrow 0$ while keeping the singular character of $\Upsilon$. This trick might be interpreted as follows: a fixed background breaks the gauge invariance of the background field method [16]. However, in order to control this breaking while keeping some gauge freedom on the background, the fields $\mathcal{F}$ and $h$ were introduced in (49). As a consequence, the functions $\mathcal{F}$ and $h$ allow $\Upsilon$ to vary through a class of backgrounds that generates (34). Then, by making $\Upsilon$ as small as possible, expression (48) reads

$$
F_{i j k}{ }^{l}(A) \rightarrow F_{i j k}{ }^{l}(A)+m^{2}\left(\delta_{i k} \delta_{j}^{l}-\delta_{i}^{l} \delta_{j k}\right) .
$$

6 Note that the covariant derivative possesses two color indices. Thus, together with the ordinary spacetime index, there will be five indices in the spacetime representation. 
And the action (16) now reads

$$
S_{\text {eff }}=\frac{1}{4 \kappa^{2}} \int \mathrm{d}^{d} x\left[F_{i j k}{ }^{l} F_{l}^{i j k}-4 m^{2} F+2 d(d-1) m^{4}\right] .
$$

We have introduced a new parameter, $m$, as a free parameter. However, this parameter is not present in the starting action. Thus, there might exist a condition to fix this parameter to a physical consistent value. For instance, the mass parameter would be such that it characterizes the vacuum nature of the background. To do so, $m$ would be fixed, in a self-consistent way, by requiring minimal dependence in $m$ of the vacuum energy,

$$
\frac{\partial \mathcal{W}}{\partial m^{2}}=0
$$

where the quantum action is defined as

$$
e^{-\mathcal{W}}=\int D A D b D \bar{c} D c e^{-S_{\text {eff }}-S_{g f}}
$$

The gap equation (52) in fact reads

$$
\langle F\rangle=d(d-1) m^{2},
$$

where $\langle F\rangle$ is the expectation value of $F$ related to the functional (53). The gap equation (54) fixes $m$ to a physical value $m_{*}$ which stabilizes the vacuum. We also remark that this kind of gap equation (52) has been frequently used in physics, in particular, in QCD where the gap equation (52) is imposed to find an optimal value for the mass gap in QCD [17]. Also in QCD, a similar gap equation is used to fix the so-called Gribov parameter, associated with the improvement of the quantization of Yang-Mills theories [18-20].

Once $m_{*}$ is determined, the existence of the background turns out to be completely characterized by $m_{*}$ and by the presence of the the color-breaking term $F$ in the action. Thus, after the computation of $m_{*}^{2}$,

$$
S_{\text {eff }}=\frac{1}{4 \kappa^{2}} \int \mathrm{d}^{d} x\left[F_{i j k}{ }^{l} F_{l}^{i j k}-4 m_{*}^{2} F+2 d(d-1) m_{*}^{4}\right] .
$$

The theory is then described by the effective action (55). We note that in the action (55) the parameters $\kappa$ and $m_{*}$ are assumed to be already fixed from usual loop quantum computations [21].

Note that, due to the color symmetry breaking mechanism, the group indices are transformed into observable spacetime indices. Thus $A_{i j}{ }^{k}$ would describe physical spin-1 excitations as well as spin-2 physical excitations.

\subsection{Gravity}

Since we now have an effective theory, where a kind of non-perturbative vacuum is consistently defined, we can perform the mapping (21) and (23), providing now

$$
S_{\mathrm{eff}}^{\mathrm{e}}=\frac{1}{4 \kappa^{2}} \int \mathrm{d}^{d} x \mathrm{e}\left[R_{\mu \nu \alpha \beta} R^{\mu \nu \alpha \beta}-4 m_{*}^{2} R+2 d(d-1) m_{*}^{4}\right]
$$

where $R=R_{\mu \nu}{ }^{\mu \nu}$.

The action $(56)$ is $G L(d, \mathbb{R})$ invariant in the spacetime sector, and, due to the presence of the EH term, we can associate it with gravity. In that sense, the mass parameter $m_{*}^{2}$ is related to both, Newton constant, $G$, and the cosmological constant, $\Lambda$, through

$$
G=\frac{\kappa^{2}}{16 \pi m_{*}^{2}}, \quad \Lambda=\frac{d(d-1)}{2} m_{*}^{2} .
$$


Thus,

$$
S_{\mathrm{eff}}^{\mathrm{e}}=\frac{1}{16 \pi G} \int \mathrm{d}^{d} x \mathrm{e}\left[\frac{d(d-1)}{8 \Lambda} R_{\mu \nu \alpha \beta} R^{\mu \nu \alpha \beta}-R+\Lambda\right],
$$

which is recognized as a generalization of the $\mathrm{EH}$ action in the Palatini formalism.

Let us derive the field equations of action (58). For the connection $\Gamma$, the field equations read

$$
\begin{aligned}
-\mathcal{D}_{\mu} R_{\beta}^{\mu \nu \alpha}- & 2\left[\frac{d}{2} Q_{\mu}+T_{\mu \kappa}{ }^{\kappa}\right] R_{\beta}^{\mu \nu \alpha}+T_{\mu \kappa}{ }^{\nu} R_{\beta}^{\mu \kappa \alpha} \\
& +\frac{2 \Lambda}{d(d-1)}\left[Q_{\beta}{ }^{\nu \alpha}-\delta_{\beta}^{\nu} \bar{Q}^{\alpha}+2 T_{\beta}^{\alpha \nu}+U_{\beta}^{\mu \nu \alpha}\left(\frac{d}{4} Q_{\mu}+T_{\mu \kappa}{ }^{\kappa}\right)\right]=0,
\end{aligned}
$$

where

$$
U_{\mu \nu \alpha \beta}=g_{\mu \beta} g_{\nu \alpha}-g_{\mu \alpha} g_{\nu \beta},
$$

again, $Q_{\mu}$ is the Weyl convector (30), which can be obtained from the non-metricity tensor $Q_{\mu \nu \alpha}$, defined as

$$
Q_{\mu \alpha \beta}=\mathcal{D}_{\mu} g_{\alpha \beta}
$$

The quantity $\bar{Q}_{\mu}$ is also a vector constructed from the non-metricity,

$$
\bar{Q}_{\mu}=Q_{v \mu}{ }^{\nu} \text {. }
$$

Finally, $T_{\mu \nu \alpha}$ is the torsion tensor (31).

The variation with respect to the metric tensor provides

$\frac{1}{2} g^{\mu \nu} R^{\sigma \rho \alpha \beta} R_{\sigma \rho \alpha \beta}-R_{\alpha \beta \gamma}^{\mu} R^{\nu \alpha \beta \gamma}-R_{\alpha \beta \gamma}{ }^{\mu} R^{\alpha \beta \gamma \nu}-\frac{8 \Lambda}{d(d-1)}\left[\frac{1}{2} g^{\mu \nu}(R-\Lambda)-R^{\mu \nu}\right]=0$.

Contraction of this equation, (63), with $g_{\mu \nu}$ results in the trace equation

$$
\frac{(d-4)}{4} R^{\sigma \rho \alpha \beta} R_{\sigma \rho \alpha \beta}+\frac{2 \Lambda}{d(d-1)}(2-d) R+\frac{2 \Lambda^{2}}{(d-1)}=0 .
$$

Let us focus on the more interesting case $d=4$. The field equations (59) and (63) now read

$$
\begin{gathered}
-\mathcal{D}_{\mu} R_{\beta}^{\mu \nu \alpha}-2\left[2 Q_{\mu}+T_{\mu \kappa}{ }^{\kappa}\right] R_{\beta}^{\mu \nu \alpha}+T_{\mu \kappa}{ }^{\nu} R_{\beta}^{\mu \kappa \alpha} \\
+\frac{\Lambda}{6}\left[Q_{\beta}{ }^{\nu \alpha}-\delta_{\beta}^{\nu} \bar{Q}^{\alpha}+2 T_{\beta}^{\alpha}{ }^{\nu}+U_{\beta}^{\mu \nu \alpha}\left(Q_{\mu}+T_{\mu \kappa}{ }^{\kappa}\right)\right]=0, \\
\frac{1}{2} g^{\mu \nu} R^{\sigma \rho \alpha \beta} R_{\sigma \rho \alpha \beta}-R_{\alpha \beta \gamma}^{\mu} R^{\nu \alpha \beta \gamma}-R_{\alpha \beta \gamma}{ }^{\mu} R^{\alpha \beta \gamma \nu}-\frac{2 \Lambda}{3}\left[\frac{1}{2} g^{\mu \nu}(R-\Lambda)-R^{\mu \nu}\right]=0,
\end{gathered}
$$

while the trace equation (64) now provides

$$
R=2 \Lambda \text {. }
$$

In the usual gravitation theory with cosmological constant, i.e., the action (58) with no quadratic terms in the curvature, the equation (66) is also obtained and the solution is the $d S_{4}$ spacetime where the curvature is given by

$$
R_{\mu \nu \alpha \beta}=\frac{\Lambda}{6} U_{\mu \nu \alpha \beta} .
$$

Remarkably, this expression is also an exact solution of (65). To see this one has to use the fact that the geometry of the $d S_{4}$ spacetime is Riemannian, $Q=T=0$. 
It is worth mentioning that one would generate a gravity theory with the $A d S_{4}$ as a solution if, in (52), a negative value for $m_{*}^{2}$ shows up. In that case $\Lambda<0$ and we are in fact facing the $A d S_{4}$ instead of $d S_{4}$.

\section{Discussions and conclusions}

In this work we have established a mapping from a Euclidean $S O(d)$ gauge theory to a dynamical spacetime with a linear connection and independent metric tensor. Moreover, in order to relate the mapping with a model for gravity, we have developed a sketch for a color symmetry breaking mechanism which generates spin-2 excitations in Euclidean spacetime. Thus, the mapping from the Euclidean space with a gauge field to a curved space with the linear connection provides a modified gravity model with cosmological constant in the Palatini formalism.

We noted that it is exactly the mappings (21) and (23) which lead action (7) into action (27). However, we strongly remark again that the presented color symmetry breaking mechanism is just a sketch. Realistic quantum computations are needed in order to give estimates for $\kappa$ and $m$ and, consequently, for $G$ and $\Lambda$. By contrast, in principle, we have a vast freedom to find suitable solutions for $\kappa$ and $m$ within the renormalization group equations. This happens because, being renormalizable to all orders in perturbation theory, the theory is scale invariant as well as renormalization scheme invariant. Furthermore, the value $m$ is related to the value of $\Lambda$ which receives several contributions from the non-perturbative vacuum energy of the rest of fundamental interactions [22]. For instance, at the electroweak sector there is a contribution coming from the Higgs vacuum while from the QCD sector there would be a contribution from the Gribov ambiguities and from condensates [20]. Obviously, even if consistent solutions might not show up analytically, numerical computations would be applied as well. Another point is that gap equation (52) is an ansatz justified by the minimization principle of the vacuum energy. It might be possible that there exists another way to compute $m$ at the quantum level. We emphasize that this kind of computation (for $\kappa$ and $m$ within the renormalization group) is beyond the scope of this paper and must be analyzed [13]. From now on, for the rest of the conclusion we will assume that the computation is possible and gives reasonable values for $m$ and $\kappa$.

Concerning the spacetime nature, we can conclude from the present proposal that, for very high energies, the fundamental spacetime would be a continuous Euclidean space. Now, once we start to decrease the energy, the background starts to show its presence, breaking the color symmetry. Thus, spin-2 physical excitations show up and the theory can be visualized as a dynamical curved spacetime. Under this scope, the fundamental spacetime configurations would be the $d S_{4}$ space (or $A d S_{4}$ if $\Lambda<0$ ).

Another point to be explored in future would be the inclusion of matter fields (nongravitational fields) in the model. In principle, we can include the standard model in this formalism since we are dealing with an Euclidean spacetime. However, it should result, at the gravitational low energy regime, in a consistent quantum standard model in a classical curved space.

An evident interpretation of the method is that there is no incompatibility between GR and QM. In fact, the quantum gravity theory is described in a Euclidean spacetime; thus, there are no problems to be faced in the space and time definitions. At the quantum level, the space and time are the usual quantum mechanical parameters. At the classical level, the space and time are translated into the GR spacetime. However, quantum effects are no longer associated with them. Thus, we can say that QG and GR would be the different sides of the same coin. 
Table 1. Quantum numbers of the relevant quantities.

\begin{tabular}{llllllll}
\hline Fields & $\partial$ & $s$ & $A$ & $c$ & $\bar{c}$ & $b$ & $\kappa$ \\
\hline UV dimension & 1 & 0 & 1 & 0 & $d-2$ & $d-2$ & $(4-d) / 2$ \\
Ghost number & 0 & 1 & 0 & 1 & -1 & 0 & 0
\end{tabular}

Finally, we recall the fact that the approach presented in this paper might be generalized to other groups but the $S O(d)$, as well as for other classes of gauge theories. For instance, one may start with a $G L(d, \mathbb{R})$ gauge theory and see if it can be cast into a dynamical spacetime. Obviously, in that particular case, the spacetime representation exists as well.

\section{Acknowledgments}

The authors would like to acknowledge A Accioly, H J M Cuesta, S A Dias, E L Rodrigues and J F Villas da Rocha for fruitful and interesting discussions. Also J A Helayël-Neto is gratefully acknowledged for fruitful and clarifying discussions, suggestions and for reviewing the paper. We would also like to kindly thank T R B M Rodrigues for suggestions in the correction of the text. Finally, the Conselho Nacional de Desenvolvimento Científico e Tecnológico (CNPq-Brazil) is acknowledged for financial support.

\section{Appendix A. Renormalizability of the model}

To study the renormalizability of the action (9), we will use the algebraic renormalization technique [14]. A basic ingredient of this method relies on the so-called BRST symmetry. For the action (9), the BRST transformations are

$$
s A_{i}=-D_{i} c, \quad s c=\frac{1}{2}[c, c], \quad s \bar{c}=b, \quad s b=0,
$$

where $s$, the BRST operator, is nilpotent $s^{2}=0$.

In order to have a consistent quantum description of the model, we introduce a set of external BRST invariant sources coupled to the nonlinear BRST variations,

$$
\begin{aligned}
S_{\text {ext }} & =s \operatorname{Tr} \int \mathrm{d}^{d} x\left(-\Omega_{i} A_{i}+L c\right) \\
& =\operatorname{Tr} \int \mathrm{d}^{d} x\left(-\Omega_{i} D_{i} c+\frac{1}{2} L[c, c]\right),
\end{aligned}
$$

where

$$
s \Omega=s L=0 \text {. }
$$

Then, in this appendix we will work with the general BRST invariant action

$\Sigma=\frac{1}{4} \operatorname{Tr} \int \mathrm{d}^{d} x F_{i j} F_{i j}+\operatorname{Tr} \int \mathrm{d}^{d} x\left(b \partial_{i} A_{i}+\bar{c} \partial_{i} D_{i} c\right)+\operatorname{Tr} \int \mathrm{d}^{d} x\left(-\Omega_{i} D_{i} c+\frac{1}{2} L[c, c]\right)$.

Let us take a look at the power counting renormalizability of (9). For that, the UV dimensions of the fields and sources are displayed in table 1. Observing the dimension of the coupling constant we see that renormalizability would be a feature of the restricted cases where $2 \leqslant d \leqslant 4$. 


\section{A.1. Symmetries and Ward identities}

The symmetries of the model are described by the following Ward identities.

- The Slavnov-Taylor identity

$$
\mathcal{S}(\Sigma)=\operatorname{Tr} \int \mathrm{d}^{d} x\left(\frac{\delta \Sigma}{\delta \Omega_{i}} \frac{\delta \Sigma}{\delta A_{i}}+\frac{\delta \Sigma}{\delta L} \frac{\delta \Sigma}{\delta c}+b \frac{\delta \Sigma}{\delta \bar{c}}\right)=0 .
$$

- The ghost equation

$$
\int \mathrm{d}^{d} x\left(\frac{\delta \Sigma}{\delta c}+\left[\bar{c}, \frac{\delta \Sigma}{\delta b}\right]\right)=\int \mathrm{d}^{d} x\left(\left[\Omega_{i}, A_{i}\right]+[L, c]\right) .
$$

- The gauge condition and the antighost equation

$$
\frac{\delta \Sigma}{\delta b}=\partial_{i} A_{i}, \quad \frac{\delta \Sigma}{\delta \bar{c}}+\partial_{i} \frac{\delta \Sigma}{\delta \Omega_{i}}=0 .
$$

- The $S L(2, \mathbb{R})$ symmetry

$$
\operatorname{Tr} \int \mathrm{d}^{d} x\left(c \frac{\delta \Sigma}{\delta \bar{c}}+\frac{\delta \Sigma}{\delta L} \frac{\delta \Sigma}{\delta b}\right)=0 .
$$

- Global color symmetry

$$
\int \mathrm{d}^{d} x\left(\left[A_{i}, \frac{\delta \Sigma}{\delta A_{i}}\right]+\left[c, \frac{\delta \Sigma}{\delta c}\right]+\left[\bar{c}, \frac{\delta \Sigma}{\delta \bar{c}}\right]+\left[b, \frac{\delta \Sigma}{\delta b}\right]+\left[\Omega_{i}, \frac{\delta \Sigma}{\delta \Omega_{i}}\right]+\left[L, \frac{\delta \Sigma}{\delta L}\right]\right)=0 .
$$

\section{A.2. Characterization of the most general counterterm}

Now, to show the renormalizability of the action (A.4), we will make use of the algebraic renormalization technique [14]. To do that, one adds to the classical action, a perturbation term,

$$
\Sigma^{(1)}=\Sigma+\epsilon \Sigma^{c}
$$

where the counterterm $\Sigma^{c}$ is an integrated polynomial on the fields and sources with the UV dimension up-bounded by four and a vanishing ghost number. The enforcement that the action (A.10) obeys the Ward identities (A.5-A.9) implies on the following constraints for the counterterm,

$\mathcal{S}_{\Sigma} \Sigma^{c}=0, \quad \int \mathrm{d}^{d} x \frac{\delta \Sigma^{c}}{\delta c}=0, \quad \frac{\delta \Sigma^{c}}{\delta b}=0$,

$\frac{\delta \Sigma^{c}}{\delta \bar{c}}+\partial_{i} \frac{\delta \Sigma^{c}}{\delta \Omega_{i}}=0, \quad \operatorname{Tr} \int \mathrm{d}^{d} x c \frac{\delta \Sigma^{c}}{\delta \bar{c}}=0$,

$\int \mathrm{d}^{d} x\left(\left[A_{i}, \frac{\delta \Sigma^{c}}{\delta A_{i}}\right]+\left[c, \frac{\delta \Sigma^{c}}{\delta c}\right]+\left[\bar{c}, \frac{\delta \Sigma^{c}}{\delta \bar{c}}\right]+\left[\Omega_{i}, \frac{\delta \Sigma^{c}}{\delta \Omega_{i}}\right]+\left[L, \frac{\delta \Sigma^{c}}{\delta L}\right]\right)=0$.

where $\mathcal{S}_{\Sigma}$ stands for the linearized Slavnov-Taylor operator,

$$
\mathcal{S}_{\Sigma}=\operatorname{Tr} \int \mathrm{d}^{d} x\left(\frac{\delta \Sigma}{\delta \Omega_{i}} \frac{\delta}{\delta A_{i}}+\frac{\delta \Sigma}{\delta A_{i}} \frac{\delta}{\delta \Omega_{i}}+\frac{\delta \Sigma}{\delta L} \frac{\delta}{\delta c}+\frac{\delta \Sigma}{\delta c} \frac{\delta}{\delta L}+b \frac{\delta}{\delta \bar{c}}\right) .
$$

The first of (A.11) defines a cohomological problem whose general solution is given by

$$
\Sigma^{c}=\Delta_{0}+\mathcal{S}_{\Sigma} \Delta^{-1}
$$

where $\Delta_{0}$ is the nontrivial part of the cohomology,

$$
\Delta_{0} \neq \mathcal{S}_{\Sigma} \cdot \text { (something), }
$$


and $\mathcal{S}_{\Sigma} \Delta^{-1}$ is the trivial part. The nontrivial part is an integrated polynomial on the fields and sources with the UV dimension up-bounded by the four and a vanishing ghost number while $\Delta^{-1}$ is an integrated polynomial with the UV dimension up-bounded by four and a ghost number given by -1 . In fact, it is straightforward to show that the most general counterterm (A.13) is determined by [14]

$$
\Delta_{0}=a_{0} S_{\mathrm{YM}} \text {, }
$$

and

$$
\Delta^{-1}=a_{1} \operatorname{Tr} \int \mathrm{d}^{d} x\left(\Omega_{i}+\partial_{i} \bar{c}\right) A_{i}
$$

where $a_{0}$ and $a_{1}$ are independent renormalization parameters.

\section{A.3. Quantum stability}

The last step to prove renormalizability is to show that the counterterm (A.13) can be reabsorbed in the classical action (A.4) by means of multiplicative redefinition of the fields, sources and parameters according to

$$
\Sigma\left(\phi_{0}, J_{0}, \kappa_{0}\right)=\Sigma(\phi, J, \kappa)+\epsilon \Sigma^{c}(\phi, J, \kappa),
$$

where

$$
\phi_{0}=Z_{\phi}^{1 / 2} \phi, \quad J_{0}=Z_{J} J, \quad \kappa_{0}=Z_{\kappa} \kappa
$$

and

$$
\phi \in\{A, b, c, \bar{c}\}, \quad J \in\{\Omega, L\} .
$$

In fact it is not difficult to show that the action (A.4) is multiplicatively renormalizable, where the independent renormalization factors are given by

$$
Z_{\kappa}=1-\epsilon \frac{a_{0}}{2}, \quad Z_{A}^{1 / 2}=1+\epsilon\left(\frac{a_{0}}{2}+a_{1}\right),
$$

and the nonindependent renormalization factors read

$$
Z_{c}^{1 / 2}=Z_{\bar{c}}^{1 / 2}=Z_{\Omega}^{-1 / 2}=Z_{\kappa}^{-1 / 2} Z_{A}^{-1 / 4}, \quad Z_{b}^{1 / 2}=Z_{L}^{-2}=Z_{A}^{-1} .
$$

This ends the proof of the renormalizability of pure $S O(d)$ gauge theory in a $2 \leqslant d \leqslant 4$ dimensional Euclidean spacetime.

\section{Appendix B. Renormalization of $m_{*}^{2}$}

In this appendix, we provide the program for derivation of the renormalization rule of the background parameter $m_{*}^{2}$. We omit the details since they are somewhat simple to perform. To do this we consider as a starting point the fact that the background is a particular configuration of the gauge field $A$. Thus

$$
\Upsilon_{0}=Z_{A}^{1 / 2} \Upsilon
$$

Now, perturbing the equation (A.17) according to (47) we will find an expression where the mass parameter $m_{*}^{2}$ is present. Thus, performing the usual multiplicative renormalization (A.18-A.19) together with

$$
m_{* 0}^{2}=Z_{m^{2}} m_{*}^{2},
$$

we find expression (35). 


\section{References}

[1] 't Hooft G and Veltman M J G 1974 Ann. Poincaré Phys. Theor. A 2069

[2] Deser S and van Nieuwenhuizen P 1974 Phys. Rev. D 10401

[3] Deser S and van Nieuwenhuizen P 1974 Phys. Rev. D 10411

[4] Stelle K S 1977 Phys. Rev. D 16953

[5] Rovelli C 2004 Quantum Gravity (Cambridge: Cambridge University Press) 455 p

[6] Smolin L 2004 An invitation to loop quantum gravity Proc. 3rd Int. Symp. on Quantum Theory and Symmetries (Cincinnatti, USA, 10-14 September 2003) (Singapore: World Scientific) pp 655-82 (Preprint hep-th/0408048)

[7] Utiyama R 1956 Phys. Rev. 1011597

[8] Kibble T W B 1961 J. Math. Phys. 2212

[9] Ferrara S 1987 Phys. Scr. T 15132

[10] Deser S 1987 Phys. Scr. T 15138

[11] Gitman D M and Tyutin I V 1990 Quantization of Fields with Constraints (Springer Series in Nuclear and Particle Physics) (Berlin: Springer) $291 \mathrm{p}$

[12] Bolivar A O 2004 Quantum-Classical Correspondence (The Frontiers Collection) (Berlin: Springer) 186 p

[13] Sobreiro R F and Vasquez Otoya V J in preparation

[14] Piguet O and Sorella S P 1995 Algebraic renormalization: perturbative renormalization, symmetries and anomalies Lect. Notes Phys. M 28

[15] Ribeiro N C, Sobreiro R F and Sorella S P 2003 Phys. Lett. A 3176 (Preprint hep-th/0302202)

[16] 't Hooft G Karpacz 1975, Proc. Acta Universitatis Wratislaviensis No 368, vol 1 (Wroclaw 1976) 345-69

[17] Sorella S P 2006 Ann. Phys. 3211747

[18] Gribov V N 1978 Nucl. Phys. B 1391

[19] Zwanziger D 1993 Nucl. Phys. B 399477

[20] Dudal D, Sobreiro R F, Sorella S P and Verschelde H 2005 Phys. Rev. D 72014016 (Preprint hep-th/0502183)

[21] Itzykson C and Zuber J B 1980 Quantum Field Theory (International Series in Pure and Applied Physics) (New York: McGraw-Hill) $705 \mathrm{p}$

[22] Shapiro I L and Sola J 2007 J. Phys. A: Math. Gen. 406583 (Preprint gr-qc/0611055) 